\title{
Thinking Establishment and Diagram Expression of Green Architecture Design
}

\author{
Zhou Zhongkai", Liu Changan, Zhao Jilong \\ School of Architecture and Urban Planning, Shandong Jianzhu University, Jinan, China

\section{Email address:} \\ zhongkai_zhou@sdjzu.edu.cn (Zhou Zhongkai),84235179@qq.com (Liu Changan), zll1-w@163.com (Zhao Jilong) \\ ${ }^{*}$ Corresponding author
}

\section{To cite this article:}

Zhou Zhongkai, Liu Changan, Zhao Jilong. Thinking Establishment and Diagram Expression of Green Architecture Design. Science Discovery. Vol. 5, No. 7, 2017, pp. 565-571. doi: 10.11648/j.sd.20170507.26

Received: November 15, 2017; Accepted: November 22, 2017; Published: December 29, 2017

\begin{abstract}
As the important way of information visualization, diagram is an efficient approach in analyzing and expressing the thinking process and achievement of green architecture design, as a result, establishing the diagram system and expression methodology could make a great contribution to optimize design process, convenient thinking communication as well as structure design methodology. Through discussion of green architecture thinking process and expression system, as a field which contains complex design elements and evaluation criteria, green architecture design needs the logic thinking and systematic expression system by applying visual diagramming language. Furthermore, through analyzing green architecture design case, this paper could further analyze the relationship among architecture design, thinking construction and diagram expression, and illustrates that diagram will offer systematic logic and methodology for the green architecture design and teaching, which finally reaches the combination between rationality and sensibility, abstraction and concretization, form and technology.
\end{abstract}

Keywords: Green Architecture Design, Design Thinking, Diagram Expression

\section{绿色建筑设计的思维构建与图解表达}

周忠凯 ${ }^{*}$, 刘长安, 赵继龙

建筑城规学院, 山东建筑大学, 济南, 中国

邮箱

zhongkai_zhou@sdjzu.edu.cn(周忠凯)，84235179@qq.com（刘长安）, zll1-w@163.com（赵继龙）

摘要：图解作为信息可视化的重要表现形式, 是对当前绿色建筑设计的构思过程和成果表达进行深化分析和直观表达 的有效方法, 构建符合绿色建筑设计操作机制和思维规律的图解系统和表达方法, 对设计过程优化、思想交流便捷化 及设计思维构建有重要的推动作用。文章通过对绿色建筑设计的构思过程和表达方式的探讨, 分析了绿色建筑设计作 为一种包含了复杂设计要素和评价标准的领域, 需要培养理性设计思维, 并运用清晰的可视化图解语言对其进行系统 化的设计表达。同时, 通过对绿色建筑设计案例的构思过程和成果表达进行系统解读和分析, 文章试图进一步研究探 讨建筑设计、思维构建和图解表达三者之间的相互关系, 阐释图解表达作为理性的思考工具, 可以为绿色建筑设计的 表达建立系统科学的思路, 并为教学实践提供一套适宜的方法, 最终达到理性与感性、抽象与具象、形式与技术的协 调统一。

关键词: 绿色建筑设计, 维构建, 图解表达 


\section{1. 引言}

伴随着当今社会发展的多元化及信息交流的复合化, 建筑设计的思考和表达方式已经由过去“简单”的技术图 纸的模式化输出, 逐渐发展为对于城市空间中各种物质要 素和人文环境的系统性、全方位解答。与此同时, 在当今 的建筑学教育和设计实践中, 设计信息的传达 (包括思考 的过程与结果) 已经从传统的语言文字中逐步解脱出来, 并与系统性的图解表达紧密相连。图解表达方式具有更直 观和简洁的形式, 可以跨越文化的障碍, 并摆脱对文字的 依赖, 因而成为设计师之间及设计师与业主之间交流沟通 最为重要的手段, 使得设计理念的传达更加清晰、更易达 成方案构思的共识并取得设计推进的动力 [1]。

作为现在和未来设计推进的重要领域, 以生态可持续 设计理念为主要特征的绿色建筑设计迅速发展, 对于建筑 设计的评价已从过去主观艺术性的感性认知, 逐步转变为 对于设计生态性的思考和可视化表达系统的理性构建。这 对传统的建筑设计操作模式提出了新的要求和挑战, 并促 使现行的绿色建筑设计在空间形态的感性创作和设计过 程的技术性展示之外, 更加注重设计思维的逻辑构建及表 达方式的理性构架。

然而, 在现代科学技术主导的时代, 当绿色建筑设计 从广义的艺术创作范畴逐步演化为高度融合技术和艺术 且相对独立的学科时, 现行的绿色建筑设计工作模式和表 达形式也暴露出某些问题和矛盾。因此, 如何从设计现象 本身出发, 将设计内在的功能性有效抽取, 根据绿色建筑 设计过程和表达的特点, 重新构架设计目标和各阶段不同 的设计内容, 建立一种逻辑性的思维秩序, 并运用图解的 手段进行理性且系统的表达阐释, 成为建筑设计教育和实 践领域所面临的重要议题[2]。

\section{2. 绿色建筑设计表达的现状问题}

绿色建筑设计, 相较于普通的建筑设计课题, 由于包 含更多生态技术要素和复杂的物质空间需求, 其生态设计 目标的确立、构思概念的提出、研究过程的推进及最终的 成果表达, 有更高的复杂性, 因此, 对整个设计系统的表 达和组织也有更高的要求。然而, 国内的建筑实践者和教 育者多是运用传统的技术性图纸 (如平面图、剖面图、空 间意向图等) 对于绿色建筑设计的过程和结论进行技术性 的绘制和表现, 难于清晰全面的传递绿色建筑设计的核心 内容, 更不要说实践中灵活运用图解思维构建设计的分析 方法[3]。在当前诸多设计案例中, 面对绿色建筑复杂的设 计目标和不同的设计阶段, 设计的图形和图像语言并未有 效表达绿色建筑的技术性内容 (如空间的物质循环、能量 计算及相关数据的量化) 和设计的创作过程, 设计创作和 表达在某种程度上未能有效衔接。

\section{1. 重感性创作而轻理性设计思维构建}

当前大多数的实际状况显示, 设计者在参与绿色建筑 设计课题的过程中, 更多的是关注美学范畴下的空间形态 设计, 而忽略了对于理性设计方法的推导和逻辑思维的构
建。尤其是当操作绿色建筑设计类的课题时, 在设计的初 始阶段完成相关调研后, 设计师习惯性的从总体布局和功 能形态入手，如同完成事先设定好的各项“规定动作”般的 推进设计, 最后再简单地将生态技术措施与建筑形体设计 叠加, 却忽略了周边环境和基地本身的生态技术条件及制 约方案发展的各项生态因子。未能通过一套逻辑的分析方 法和思维框架, 将各项与生态技术指标相关的制约因素逐 步、合理、有序地渗透融入到建筑设计的不同阶段, 使得 看似完整的设计成果, 更多的是对于设计片段的图形化展 现, 缺少对于设计过程的总体组织及清晰阐释, “只见树 木，不见树林”。

\section{2. 图象表现而非图解表达}

计算机技术的发展和工业化生产的介入, 带来了标准 化的设计和制造规范，流水线式的生产模式削弱了了绿色 设计过程中“创作”的分量。设计人员对于绿色建筑设计的 表达更多的是“程式化”输出, 却未能将设计构思纳入到一 种逻辑化的图解语言中。有时, 设计思维的产生与表达内 容无关, 对于绿色建筑设计的内容传达, 设计者往往关注 于图纸版面的视觉效果, 通过逼真的效果图、精确的平立 剖面图等营造出令人兴奋的三维空间图景。对于创作和思 考过程的阐释, 未能通过直观的图解方式进行展现, “花 拳绣腿” 的图形掩盖了设计的实质内涵, 设计内容的传达 变成了流于表面的形式游戏, 而非源于设计本身的理性思 考。

\section{3. 思考与操作方法}

绿色建筑的设计过程, 除满足传统建筑类型的空间美 学、功能组织及人文环境等要求外, 同时要加入气候条件 （如风和日照）、环境指标（温度和湿度）、材料特性等 诸多因素作为评价标准, 有着更为复杂的设计过程。因此, 对于绿色建筑设计的思考和表达, 需要灵活结合其“生态” 特性, 并基于不同阶段的内容要求和设计目标, 逐步建立 一套针对绿色建筑设计的思考创作方法, 架构出符合绿色 建筑设计表达特点的图解系统。同时, 探讨图解表达在绿 色建筑设计的各个阶段的运用--包括设计概念、生态技术 系统、研究过程及最终空间效果的可视化表达, 总结其中 的思考逻辑和操作过程, 最终构建清晰的设计逻辑、形成 理性的图解思维模式[4]。

具体来说，在绿色建筑设计的不同阶段，经过篮选不 同的图解表达介质、提炼其内在逻辑, 进而定义与其表达 方式相对应的绿色建筑设计内容。首先, 在绿色建筑设计 的前期构思和概念生成阶段, 根据设计立意在宏观层面 (如城市设计) 和微观层面 (建筑及场地本身) 的不同定 位, 运用二维或三维图形将核心理念简练、明确、清晰的 展现, 表达出设计与场地环境、交通、气候等因素之间的 相互影响关系 [5]。其次, 在设计的深化发展阶段, 运用解 析性的图解对绿色生态技术的选用、多方案比选、形体空 间的演进、功能组成、绿色建筑技术与方案空间设计的整 合等设计内容进行形象直观的表达。最后, 在最终设计成 
果的展示阶段, 图解方式将传统的技术性图纸 (如平面图, 立面图, 剖面图等) 通过更为直观生动的形式 (如剖透视, 多层级的炸开图) 进行表达, 同时将物质循环和能量转换 等生态设计关系在技术性图式中进行细致表达 [6]。

绿色建筑设计, 以一个逻辑的思维系统作为框架, 串联各部分内容, 通过图解这一介质进行系统的可视化 传达, 可以不断地启发设计思维并有助于完善整个设计 思维框架的构建, 更好的表达绿色建筑功能形态、环境 场地及技术可持续性之间的相互关系[7]。

\section{4. 设计案例探索}

对于绿色建筑设计的思维构建及图解表达的探索和 思考, 需要在相关设计案例中进行讨论和完善。在此, 以 “生产性要素融入的绿色社区设计”这一绿色设计课题为 例, 探索并讨论绿色建筑设计的思维 (分析) 体系构建和 图解思维的设计表达这两个方面。在此次设计课题的训练 中, 我们试图启发并引导参与者首先设定设计思维框架, 按照一定的逻辑合理构架概念提出、深化及最终成果等各 个阶段。设计的思考和操作过程通过图形和图像的图解方 式进行理性展现, 绿色技术和生态因素被有机的融入到建 筑的功能空间设计中，贯穿整个设计过程。由于受到客观 条件 (如数据信息的获取渠道) 及自身认知水平所限, 设 计思维的逻辑构建和表达过程不免存在问题和不足, 但对 于绿色建筑设计的分析方法和表达过程仍然具有一定的 启发和指导意义。

题目被设定在处于济南市东部新城的山东建筑大学 新校区, 与急速扩张的城市区域相似, 随着学校办学规 模的扩大, 目前也面临着学生宿舍及餐厅空间使用日趋 紧张, 基础设施不足的状况。因此, 设计任务拟定在现 有学生生活区东侧一块约 3.46 公顷的规划用地上, 拟建
总建筑面积为 40000 平方米 (地上 35000 平方米, 地下 5000 平方米) 的学生宿舍、青年教师公寓及食堂等服务设施 (图1)。在满足功能使用要求的前提下, 如何以建筑和 景观为物质载体、城市农业生产为媒介、物质循环利用 为技术手段, 构建一个人与自然和谐共生, 融生产、生 活、生态为一体的创新绿色校园生活社区，从形体空间 和景观生态角度入手缓解校园问题, 是本次研究性设计 课题的主要目的所在。

\section{1. 设计总体框架}

与一般的建筑设计课题经过实地调研后直接进入空 间形体设计，最后再结合生态技术的线性设计模式不同。 设计者在初始阶段便被要求结合现状空间、功能、环境及 生态技术等多方面要素, 设定出总体的设计策略和思考框 架, 以理性的分析方式引导设计的推演。同时, 在设定的 总体思维框架内, 设计内容仅被模糊地定义, 在随后的设 计过程中, 运用图解方式和量化对比等手段不断调整优化 设计结构, 从多种可能的设计图景中明确并完成最终的设 计成果（图2）。

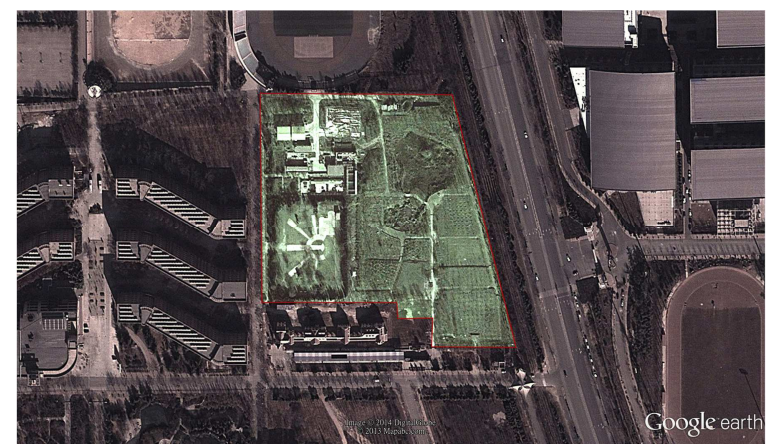

图1 基地现状。

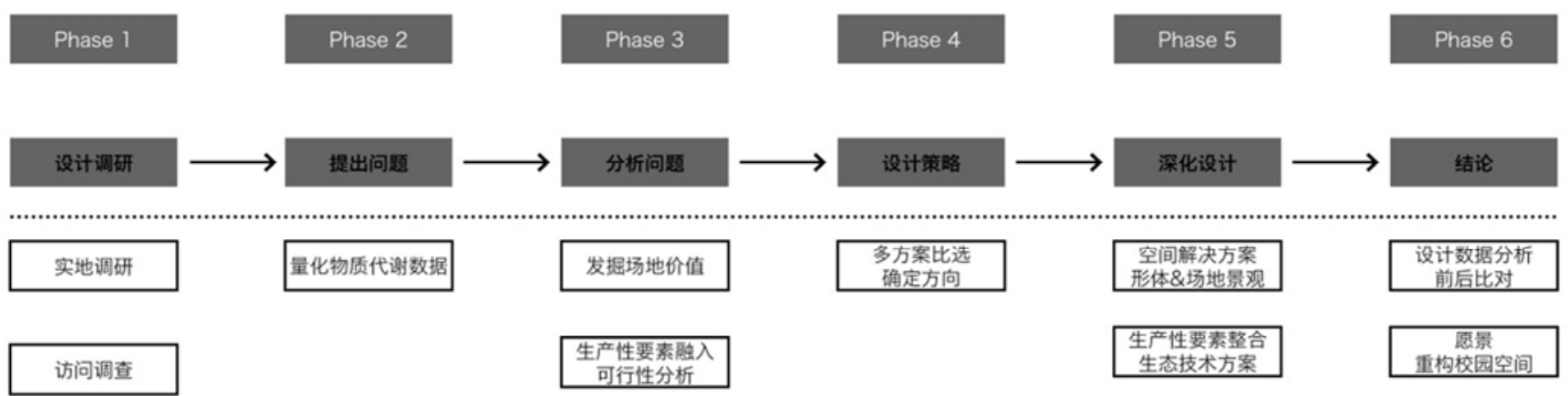

\section{资料查阅}

图2 设计框架。

\section{2. 设计前期一问题整理与信息收集}

对于设计问题的收集是整个设计过程的开始，也是 图解思维运用的起始阶段, 为明确设计目标和方向进行 铺垫。利用恰当的图解将项目的社会背景进行抽象化展 示, 并将调研所获取的与生产性相关的各项技术数据, 如 水、能量、食物消耗, 结合现状的物质输入输出模型绘
制为空间分布及流动体系图, 单一的数据信息借助生动 的图示进行解读[8]。同时, 基地周边的功能业态、交通 使用方式和气候因子等物理信息的图示化描绘, 形象而 充分的表达了设计场所的环境特征及人员活动方式和密 度, 为下一步设计策略的提出准备了充分条件（图3）。 


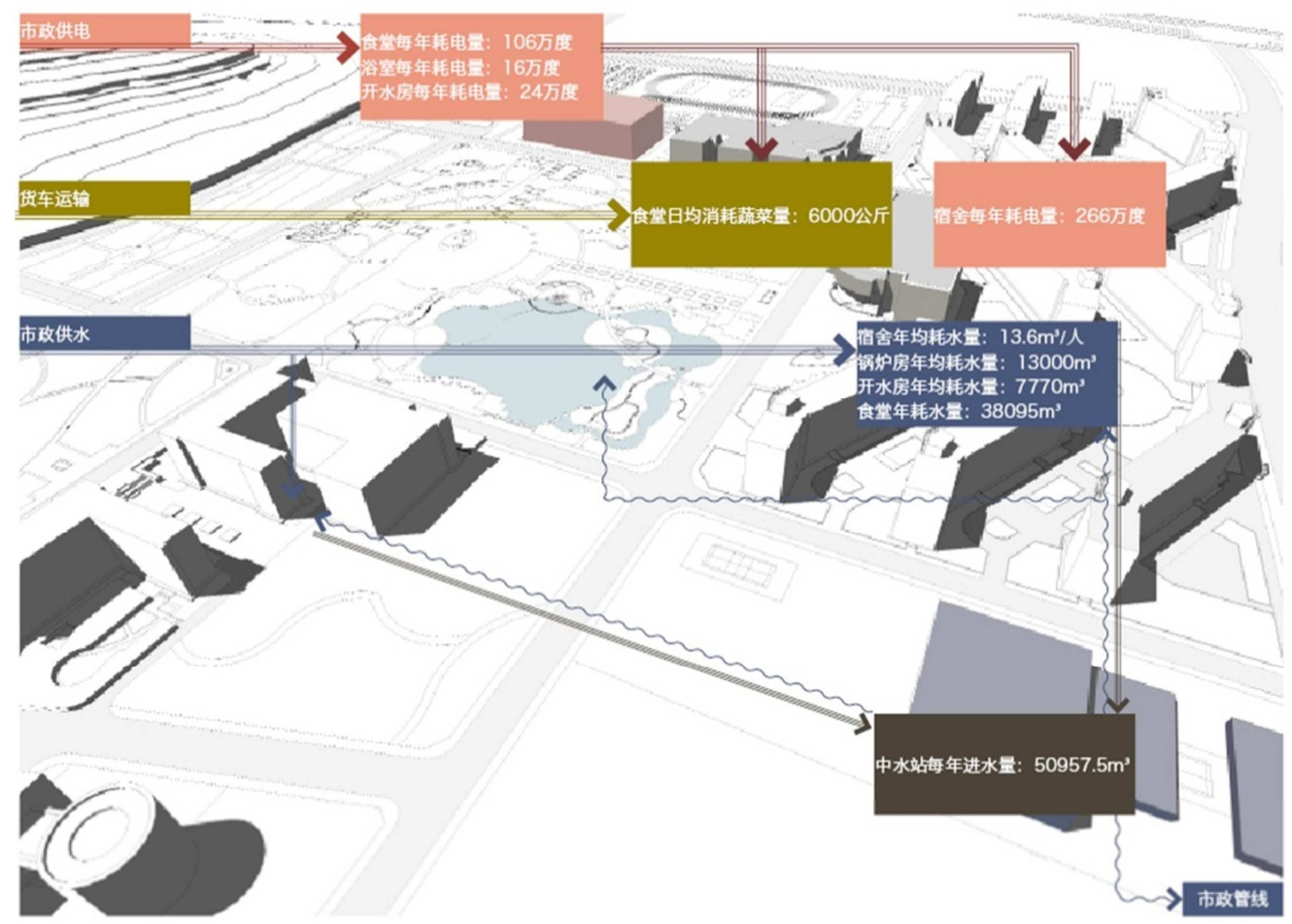

图3 基地分析。

\section{3. 初始设计--整体策略的确立}

在理解场地空间特征和各项环境要素现状的基础 上, 逐步清晰了对于空间布局和生产要素介入的整体设 计策略：以“最小性接入”原则, 基于场地现状和未来可 能的功能需求, 在各物质要素之间建立循环互动的生态 模式（图4）。首先, 根据不同种植方式的产出和用地 密度比例关系, 确定出较为理想的温室种植与传统耕地 的种植面积比例为 4:1, 作为场地农作物生产面积的设
计参考（图5）。其次，在任务书规定的容积率1、建筑 密度不大于 $30 \%$ 的条件下, 根据建筑整体在不同平均层 数下的场地占用状况, 得出建筑层数与占地密度的直观 表达图, 以此作为确定场地内建筑体量和密度形态的重 要参考（图6）。设计目标的数据内容被转译为直观的 可视化图解信息, 结合设计目标的确立, 通过比选的手 段确定了相对优化的空间使用方式。

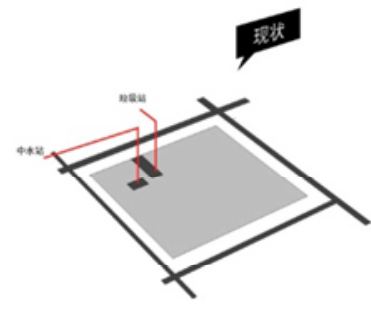

基地现有设店 : 垃圾站，中水站

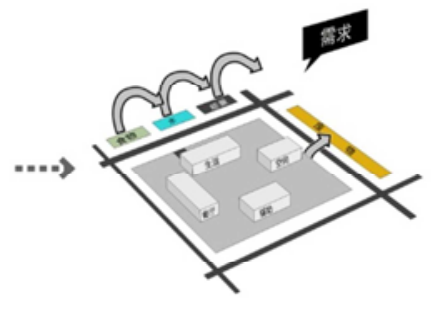

物质、能量、水赫入空间，产生废物

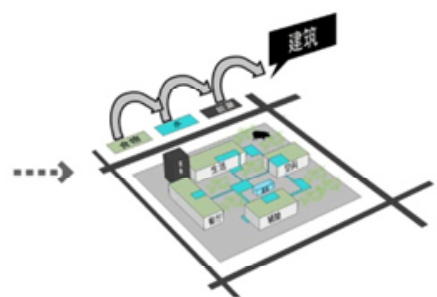

零求项目介入空间，并与建筑结合

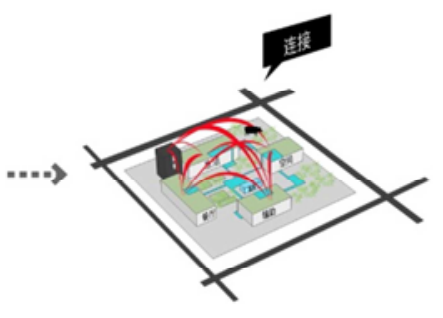

建筑空间与需求之间的联系密切

图4 设计策略。

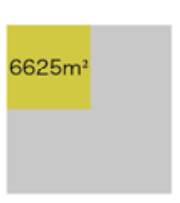

温室产量: 耕地产量

5: 0

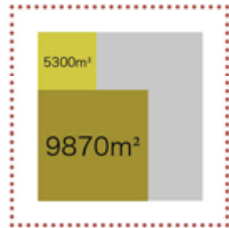

4: 1

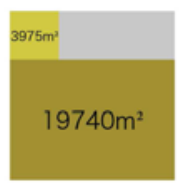

3: 2

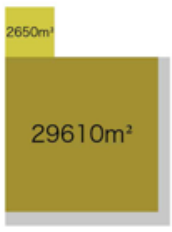

2: 3

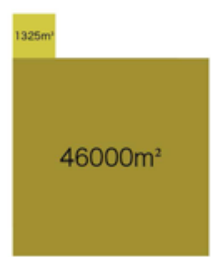

1: 4

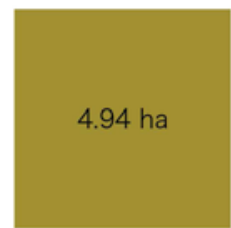

$0: 5$

图5 种植方式与产出关。 


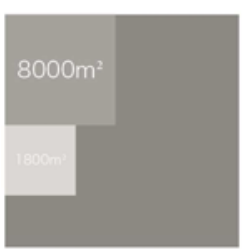

建筑平均层高

IF

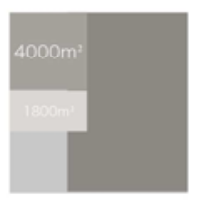

$2 \mathrm{~F}$

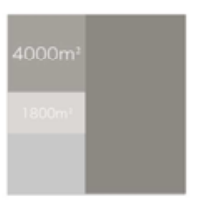

3F

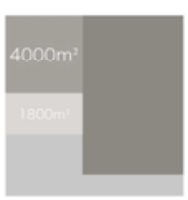

$4 \mathrm{~F}$

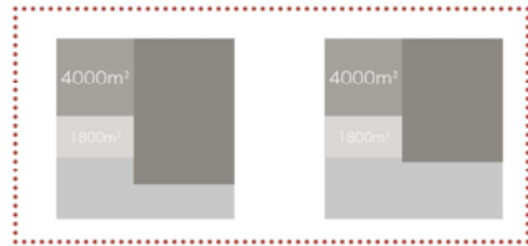

$5 \mathrm{~F}$

$6 \mathrm{~F}$

图6 建筑层高与密度。

通过综合考虑周边场地功能的临近性、人员的活动方 式、场地坚向特征及日照风向等因素，发掘各类要素和未 来空间设计联系的可能性, 推导出方案的用地策略和整体 布局方式（图7）。作为解决问题的出发点，此时脑中的
策略是模糊的, 图解表达的信息往往包含了更多的主观因 素和个人经验成分（比如功能的空间布局方式）。然而, 初始构思阶段的图解信息, 除了清晰的表达之外, 更多的 是激发创作思维, 形成抽象化的方案构思原形。
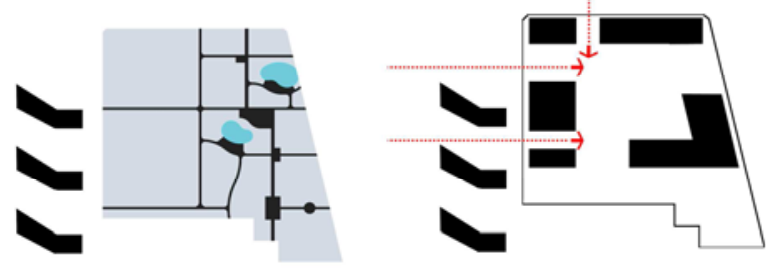
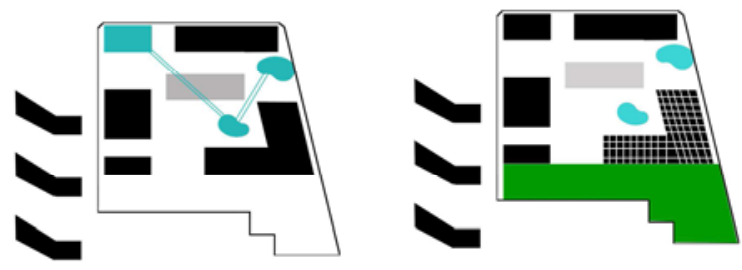

图7 总平面场地设计策略。

\section{4 . 深化设计与评价验证}

确立总体设计策略之后, 需要从空间形态、场地景观 及技术整合三个方面深化设计, 以明确各组成要素在整个 生态设计构架中的相互关系。在这一过程中, 基于前期量 化的总体布局方式和种植方案, 运用可以表达设计过程和 逻辑结构的解析性图解内容, 展现各种场地元素和功能技 术之间的相互关系。
空间形态与景观策略: 根据推演出的建筑体量密度参 考, 经过多个方案的比选, 确定以不同形态的“合院空间” 作为基本元素, 在水平和垂直界面组织各功能分区及其内 部空间, 形成丰富多变、联系紧密、易于交流的功能形态, 同时，农业生产被引入传统的地面及屋面绿化，并与原有 的中水处理设施结合, 绿植屋顶界面与地面以坡地形式自 然连接, 形成具备生产性的连续生态水体景观。空间形态 的推敲过程以一系列生成性的过程化图解进行展现和解释, 强调了构思的初始动机和最终形态之间的转形关系 (图8)。

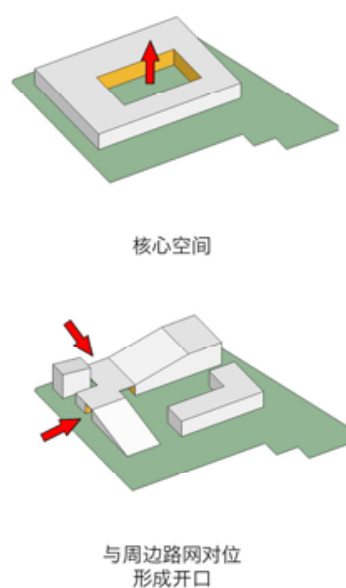

形成开口
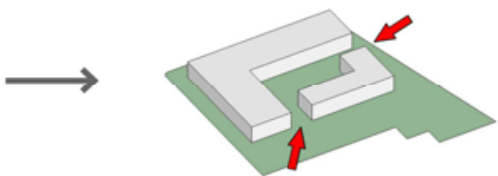

内外联通

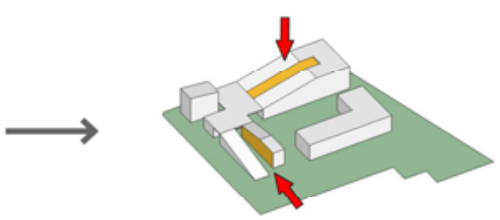

生成内院

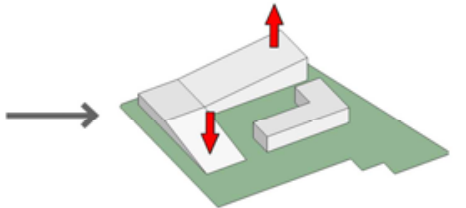

坡地链接屋面与地面

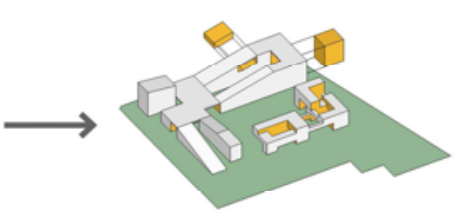

活动空间

图8 形体推演。

技术整合策略: 技术在这里不再是游离于主体设计方 案之下的边缘要素, 通过能量循环、水分循环、物质循环 及生产性种植技术等方面的阐释, 与生产性要素相关的生 态循环技术与功能空间被融合为均衡有序的整体。建筑及
景观场地的生态价值在适宜的技术措施介入下, 空间潜力 得以挖掘和拓展, 并通过完整的循环体系图清晰的标示出 各生态要素和建筑场地在空间上技术关联性（图9）。 


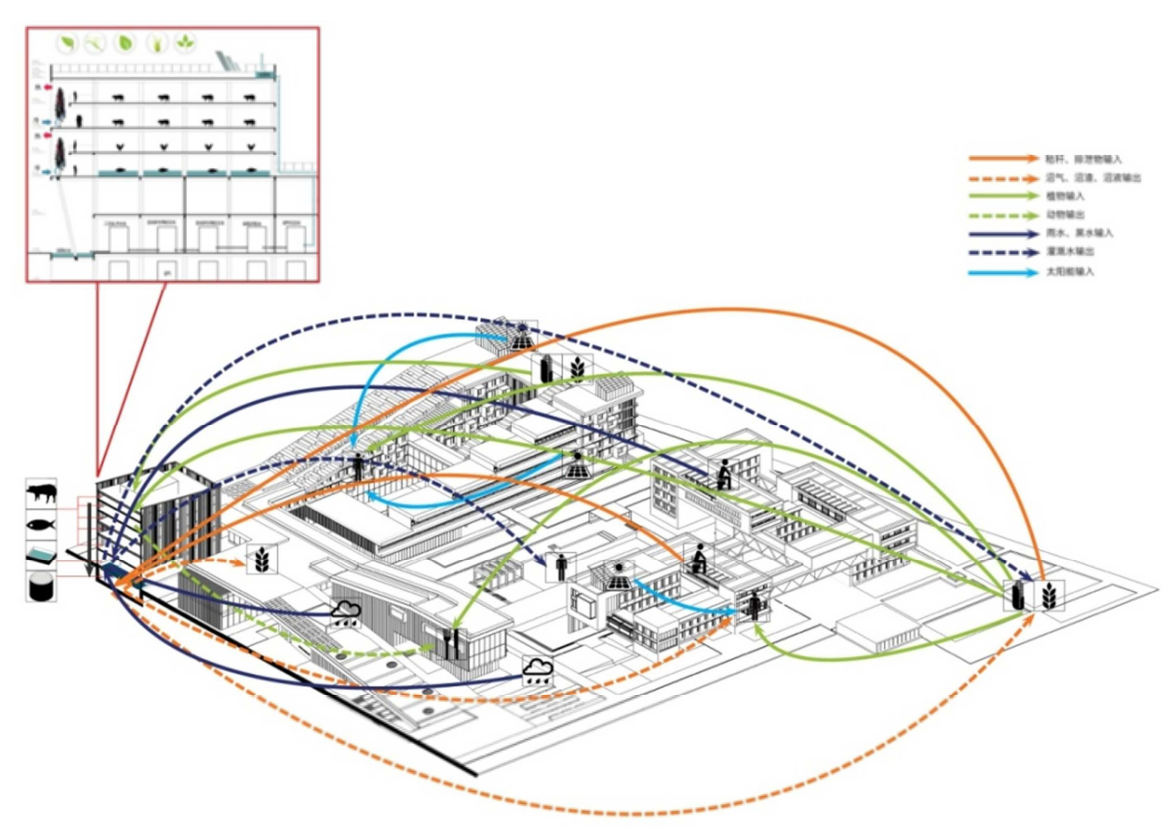

图9 物质代谢分析。

设计评价与验证: “量化”理念再一次被运用, 并通过 “物质循环体系图”完整的展现了方案中各种生态物质元 素在空间中的相互连接和循环关系, 继而进一步阐释了作 为技术和空间高度整合的生态设计方案, 在现状基地状况
和理想的运转条件下能够达到的最大物质输出量 (图10)。 同时, 与现状的物质输入输出进行比对, 强调了有机整合 后的“生产性要素接入的绿色社区”在满足空间功能布局 的前提下, 具有较好的生态性和生产效率。

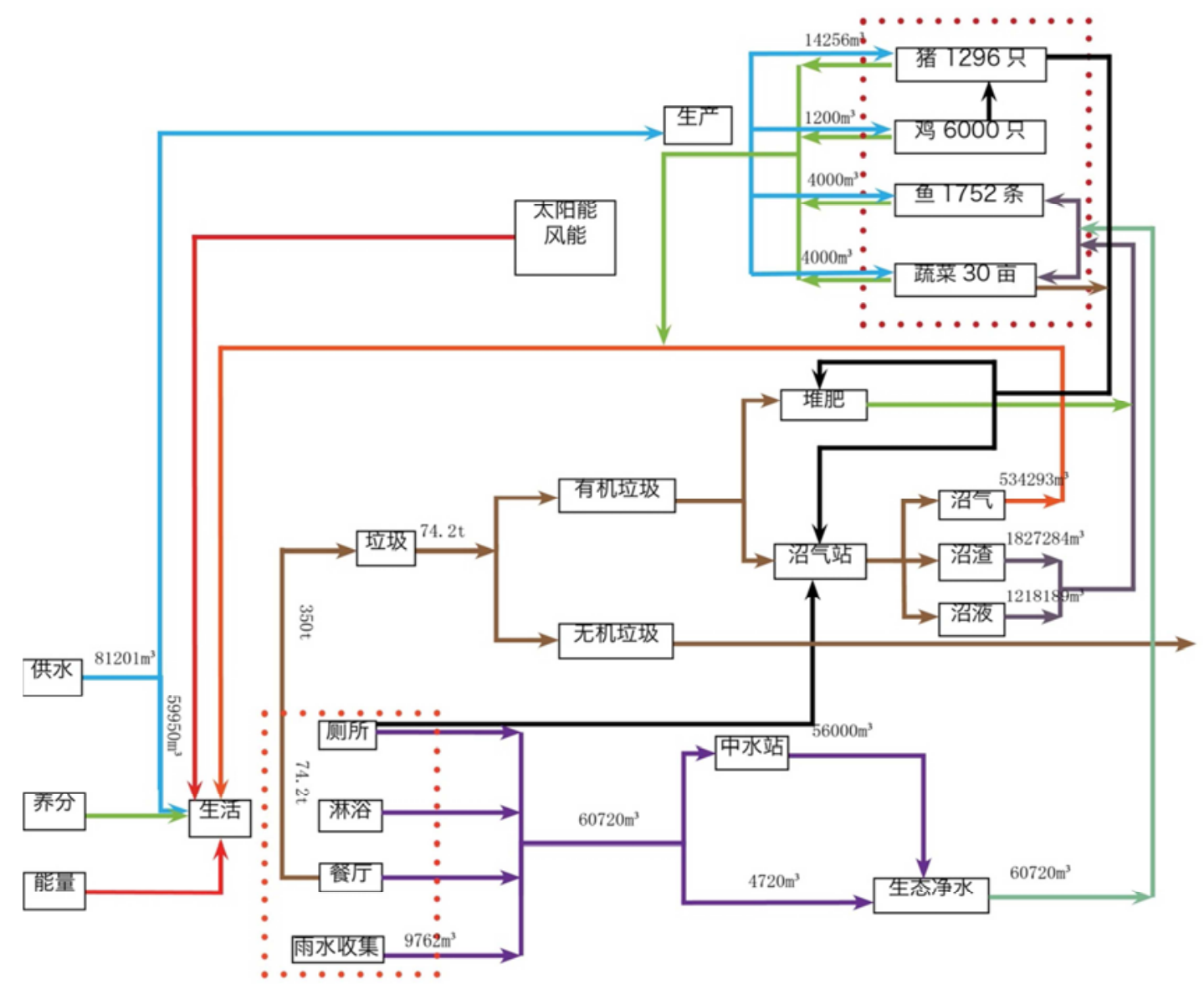

图10 物质循环流程图。 


\section{5. 结论}

“授人以鱼，不如授之以渔”。建筑设计是一门涉及多 个学科知识的综合学科, 绿色建筑设计作为其重要的发展 方向, 在传统的人文空间和功能美学训练评价体系之外, 融入了更多的绿色技术内容和生态气候目标。因此, 在绿 色建筑设计教学实践中, 迫切需要针对其多元化的复杂性 特点, 进一步研究探讨建筑设计、思维构建和图解表达三 者之间的相互关系, 以设计知识作为基础, 运用图解语言 组织设计构思过程和表达方式, 以更好的传达和阐释绿色 设计的理念及分析过程[9]。在某种程度上, 也为建筑学专 业实践探索一条系统的、理性的思路, 为专业教育, 尤其 是绿色建筑设计的教学实践提供一套适宜的方法, 最终达 到理性与感性、抽象与具象、形式与技术的协调统一 [10]。

\section{致谢}

本文为国家自然科学基金面上项目《基于养分循环代 谢的城市有农社区空间、技术、社会互动机理及构建模式 研究》(项目批准号：51778349)的阶段性成果之一。

\section{参考文献}

[1] 张琪琳, 韩冬青. 图解一期待未知. 新建筑 [J], 2008(2):118-124。
[2] 胡友培, 丁沃沃. 彼德·艾森曼图式理论解读——建筑学图 式概念的基本内涵 [J]. 建筑师，2010,146(4):21-29。

[3] 胡友培, 丁沃沃. 安东尼. 维德勒图式理论解读——当代城 市语境中的建筑学图式[J]. 建筑师, 2010,147(5):5-13。

[4] [丹麦]布莱恩. 劳森著; 范文兵译. 设计思维-建筑设计过程 解析 $[\mathrm{M}]$. (知识产权)专利文献出版社, 2007 。

[5] 安托万. 皮孔, 周鸣浩. 建筑图解, 从抽象化到物质性[J]时 代建筑, 2016(5):14-21。

[6] Martin J. Eppler, Sebastian Kernbach. Dynagrams. Enhancing Design Thinking Through Dynamic Diagrams [J]. Design Studies, 2016(47):91-117.

[7] 金熙, 沈守云, 高波. 量化思维在绿色建筑设计教学中的应 用研究 [J]. 中南林业科技大学学报 (社会科学 版. 2012,6(3):149-152。

[8] 吴锦绣, 陈晓阳, 张慧. 绿色建筑设计理念在教学中的应 用 [J]. 建筑学报，2011(11):95-97。

[9] Berkel B V. Bos. 图解 [J], 刘延川译. 建筑创作 [J], 2006(8):146-149。

[10] 蒙小英. 基于图示的景观图式语言表达 [J]. 中国园林, 2016(2):18-24。 